\title{
IMUNIZAÇÃO E SUA HISTÓRIA SOB AS LENTES DA ENFERMAGEM
}

Fernanda Batista Oliveira Santos' ORCID: 0000-0002-8523-0547

Maria Angélica de Almeida Peres" ORCID: 0000-0002-6430-3540

Deíse Moura de Oliveira"I ORCID: 0000-0003-3804-7413

Cecília Maria Lima Cardoso Ferraziv ORCID: 0000-0001-6897-3798

Rafaela Siqueira Costa Schreck' ORCID: 0000-0001-5251-3973

Universidade Federal de Minas Gerais. Belo Horizonte, MG.

"Universidade Federal do Rio de Janeiro. Rio de Janeiro, RJ.

"'Universidade Federal de Viçosa. Viçosa, MG.

IVFaculdade Ciências Médicas de Minas Gerais. Belo Horizonte, MG.

Autora Correspondente: Fernanda Batista Oliveira Santos E-mail: fernandabosufmg@gmail.com

Como citar: Santos FBO, Peres MAA, Oliveira DM, et al. Imunização e sua história sob as lentes da Enfermagem. In: Silva TMR, Lima MG, (Orgs.). Estratégias de vacinação contra a COVID-19 no Brasil: capacitação de profissionais e discentes de enfermagem. Brasilia, DF: Editora ABen; 2021. P 21-30. (Série enfermagem e pandemias, 6). https://doi.org/10.51234/aben.21.e08.c03

Revisora: Flávia Bravo. Presidente da Sociedade Brasileira de Imunizações.

\section{INTRODUÇÃO}

"A enfermagem é como a espinha dorsal do sistema de saúde". Margareth Chan/Organização Mundial da Saúde

Uma produção textual acerca da história da imunização conduzida por um grupo de cinco enfermeiras parece ser uma ideia socialmente aceitável, afinal a enfermagem é reconhecida como um campo profissional que se dedica à saúde pública brasileira e mundial. No entanto, ao revisitarmos a literatura disponível ${ }^{(1-2)}$ para tecer os principais pontos sobre a história da imunização, encontramos uma historiografia que não tem como líderes os enfermeiros, tão pouco são eles os protagonistas nos escritos desse campo do conhecimento.

Foi pensando em minimizar esse hiato nos registros da história que nos debruçamos neste capítulo, detendo-nos ao foco discursivo da imunização e sua história sob as lentes de quem tanto se dedica ao processo de imunização: a enfermagem.

Precisamos pontuar, ao assumir a enfermagem como protagonista deste capítulo, que não deixamos de reconhecer que é na força conjunta da equipe multiprofissional e no saber interdisciplinar que estão as oportunidades de quebra de paradigmas de um modelo de cuidado de saúde centrado na figura única de um profissional, que sozinho não responde às necessidades populacionais. $\mathrm{O}$ Sistema Único de Saúde (SUS) é assertivo sobre a necessidade de investimento prioritário na promoção da saúde e prevenção de doenças para manter uma população saudável. Nesse sentido, pensando no tempo presente, a imunização é essencial para um bom planejamento das ações de saúde pública.

Assumimos que o Programa Nacional de Imunização (PNI) é um dos grandes marcos históricos para o protagonismo da enfermagem brasileira nessa discussão. A partir 
desse entendimento de marco, traçamos a história possível do ontem com uma problematização em torno das enfermeiras de saúde pública com formação no modelo anglo-americano adotado no país na década de 1920; do hoje beligerante, em razão do contexto da pandemia do COVID-19, que mais uma vez colocou a enfermagem em alta visibilidade para apoio da crise sanitária com sua importante atuação na vacinação.

Ao colocarmos a enfermagem como um dos grupos protagonistas na história da imunização, buscamos fazer um movimento de reconhecimento de uma classe que tem feito, de forma mais evidente nesse período de pandemia de COVID-19, a história da imunização da civilização humana, mas sem documentá-la sob o seu ponto de vista, sob a sua concepção e sob seu olhar histórico, certas de que essa obra, guiada pela ciência e pelo compromisso ético, também se constitui em uma importante contribuição para a profissão e para o cenário da saúde, sobretudo da saúde pública brasileira.

\section{BREVE RESGATE DA HISTÓRIA DA VACINA NO MUNDO}

Para entendermos o papel central da enfermagem na imunização ao longo da história, buscamos os contextos que delinearam as primeiras formas vacinais no mundo e no Brasil. Acreditamos que, dessa forma, ficará ainda mais claro ao leitor a nossa afirmação da necessidade de a enfermagem assumir nos registros o seu relevante papel nessa ação de saúde pública.

Nesse sentido, vale pontuar que o surgimento da primeira forma de vacinação no mundo se relaciona com uma das piores epidemias da humanidade. No início do século XVII, a varíola era uma das doenças infectocontagiosas mais transmissíveis do mundo, com alta letalidade. Estima-se que tal doença tenha causado a morte de mais de 300 milhões de pessoas, durante os 80 anos em que o vírus transmissor da doença esteve circulando nos diversos continentes, superando, em número de mortes, doenças como a peste, hanseníase, gripe espanhola, tuberculose e Aids ${ }^{(1,3)}$.

Diante desse cenário, medidas urgentes de controle e erradicação da varíola fizeram-se necessárias. Em 1796, o médico inglês Edward Jenner iniciou as primeiras investigações que, posteriormente, dariam origem às técnicas de imunização. Jenner observou que um grande número de pessoas se mostrou imune à varíola. Todas realizaram ordenha do leite de vacas e tinham se contaminado com cowpox, uma doença bovina semelhante à varíola pela formação de pústulas, mas que não causava a morte dos animais. Após uma série de experiências, constatou que estes indivíduos se mantinham refratários à varíola, mesmo quando inoculados com o vírus. $A$ partir dessa observação, o médico inglês passou a extrair a secreção das lesões dos agricultores e a inocular em pessoas susceptíveis a varíola, comprovando que o material inoculado garantia proteção à doença ${ }^{(4)}$.

Essa metodologia passou a ser utilizada para imunizar contra a varíola, tendo Jenner nomeado esse material de vacina, uma derivação da palavra vaca em latim. Em 1798, Jenner publicou suas observações no trabalho Variolae Vacinae, o que causou resistência no universo médico conservador daquele momento. Mas, as comprovações eram inegáveis e, em 1840, o governo britânico instituiu a técnica de Jenner como a única forma de prevenção para os casos de varíola, fazendo com que o médico ganhasse notoriedade ${ }^{(1,4)}$.

A relação causa-efeito entre a presença de microrganismos patogênicos e a manifestação de doenças foi estabelecida anos mais tarde, aproximadamente, em 1870, pelo cientista francês Louis Pasteur e pelo médico Robert Koch. Posteriormente, Pasteur introduziu metodologias laboratoriais para descobrir a vacina contra cólera aviária e raiva humana, designando com o nome de vacina qualquer preparação utilizada para imunização de uma doença infecciosa: uma homenagem às primeiras descobertas de Edward Jenner ${ }^{(1,3)}$.

A inovação do processo da técnica de cultivo do vírus em embrião de pinto garantiu mais estabilidade para a vacina de varíola. Em 1959, a Organização Mundial da Saúde (OMS) estabeleceu um programa global para a erradicação da varíola, que financiou a produção de imunizantes da doença. Em 8 de maio de 1980, na 33ª Assembleia Mundial da Saúde estabeleceu-se o primeiro caso de erradicação global de uma doença, ao declarar que "o mundo e todas as pessoas estavam livres da varíola"(4-5). 
Desde o advento da vacina de varíola, o enorme avanço científico e tecnológico na área da biologia molecular contribuiu para que vários estudos fossem realizados, em todo o mundo. Dessa forma, foram produzidos imunobiológicos no combate a diversas doenças infecciosas, como, por exemplo, vacina combinada de tétano, difteria e coqueluche; tuberculose, febre amarela, sarampo, poliomielite e influenza $a^{(1-3)}$.

Atualmente, países desenvolvidos e com elevado crescimento econômico e social lideram empresas farmacêuticas multinacionais que realizam grandes investimentos em estudos e produção de imunobiológicos. As expectativas são por imunizantes cada vez mais eficazes e com efeitos adversos reduzidos, que poderão trazer aumento na expectativa e qualidade de vida da população global ${ }^{(6)}$.

Em contrapartida, países subdesenvolvidos e em desenvolvimento enfrentam dificuldades para acesso à tecnologia de produção de imunobiológicos com poucos investimentos governamentais. Além disso, esses países encontram-se em desvantagem no consumo das vacinas produzidas em outras localidades, devido ao alto custo e pequena escala de produção, com distribuição prioritária para os países produtores(1).

Ainda que de forma incipiente, um dos Objetivos de Desenvolvimento Sustentável (ODS), referente à saúde, incorpora a cobertura universal de vacinas nos sistemas de saúde, incluindo a necessidade de acordos internacionais entre países fornecedores e consumidores dos imunobiológicos ${ }^{(7)}$.

Para a continuidade da construção de uma história da vacinação que seja equânime, com imunização eficaz para a saúde pública global, são necessárias medidas de financiamento, regulamentação e governança que viabilizem a incorporação de amplos programas de imunização. Essas medidas fazem-se ainda mais necessárias diante do cenário atual crítico da pandemia de COVID-19, em que desafios estruturais agravam ainda mais as desigualdades no acesso à vacinação universal.

\section{PRINCIPAIS MARCOS DA HISTÓRIA DA VACINA NO BRASIL}

A história da vacina no Brasil converge com a epidemia mundial de varíola. Registros documentais descrevem que, em 1804, o militar conhecido como Marquês de Barbacena enviou sete escravos para a Europa para serem inoculados e então trouxeram os vírus vaccínia infectados no braço, seguindo a técnica de imunização do médico Edward Jenner ${ }^{(1)}$.

Em 1811, para conter a disseminação da virose e ampliar a vacinação, Dom João criou a Junta Vacínica da Corte, que, para reforçar a prática da vacinação, foi transformada, em 1846, em Instituto Vacínico do Império, afirmando o caráter obrigatório da vacinação da varíola. No entanto, a eficácia da vacina continuava a ser criticada e a obrigatoriedade da vacinação não foi efetivamente cumprida(1).

No período de 1900-1901 foram criados no país o Instituto Soroterápico do Rio de Janeiro, com direção do médico Oswaldo Cruz, e Instituto Serumtherápico, com Vital Brazil, em São Paulo, que se dedicavam à produção da vacina para varíola e dos soros contra a peste bubônica e difteria. Essas instituições dariam origem às atuais, respectivamente, Fundação Oswaldo Cruz (Fiocruz) e Instituto Butantan ${ }^{(8)}$.

Em 1904, no Rio de Janeiro, houve uma disseminação sem controle da varíola, com grande número de mortes. Diante disso, o sanitarista Oswaldo Cruz, então Diretor de Saúde Pública, encarregado de controlar as epidemias no Brasil, conseguiu a aprovação da lei que instituiu a vacinação obrigatória ${ }^{(1-4)}$.

A obrigatoriedade da vacinação, associada a fatores políticos e sociais da época, gerou uma reação popular contrária que ficou conhecida como a "Revolta da Vacina" e marcou a história da saúde pública brasileira. Para os opositores da vacinação, a obrigatoriedade era uma clara violação da liberdade individual e muitos acreditavam que a própria vacina ajudava a propagar a doença ${ }^{(4,9)}$.

Durante a Revolta da Vacina, milhares de pessoas saíram às ruas do Rio de Janeiro para protestar em um enorme conflito com 30 mortos, 110 feridos, 945 detidos e centenas de deportados. Esse levante popular teve como consequência o fim da vacinação obrigatória, decretada pelo então Presidente da República Rodrigues Alves ${ }^{(9)}$. 
Posteriormente, a vacinação continuou sendo realizada e foi sendo incorporada lentamente ao cotidiano da população da capital e das principais cidades do país, com queda, em 1906, da mortalidade por varíola, para praticamente zero. Tal fato aumentou o prestígio social de Oswaldo Cruz, que passou a ser reconhecido pelas suas ações em prol da saúde pública ${ }^{(4,9)}$.

Em 1927, iniciou-se a vacinação contra a tuberculose com a vacina BCG (Bacilo de Calmette e Guérin) no Brasil. Desde o fim da década de 1930, a vacina para a febre amarela passou a ser produzida e distribuída como foco das ações da saúde pública no país com eliminação da forma urbana da doença em $1942^{(9)}$.

Até a década de 1950, as respostas do Estado Republicano ainda eram restritas às ações emergenciais para enfrentamento de surtos epidêmicos de forma insuficiente e descontínua. Com exceção das vacinas de febre amarela e BCG, de competência do governo federal, alterações para ampliação da cobertura vacinal dependiam de governos estaduais e locais ${ }^{(1,4)}$.

Em 1959, a resolução da XI Assembleia Mundial da Saúde sobre "a necessidade e urgência de uma campanha global de erradicação da varíola" teve repercussões importantes e, em 1966, foi criada a Campanha Nacional de Erradicação da Varíola. Os desafios para organização de um programa de imunização foram expressivos como falta de consenso entre as autoridades da saúde pública e os médicos, e dificuldades para distribuição e aplicação das vacinas. A despeito das dificuldades enfrentadas, graças à vacinação em massa, em 1973, o Brasil recebeu a certificação internacional de erradicação da varíola ${ }^{(1,9)}$.

Nesse mesmo ano, foi formulado pelo Ministério da Saúde, o Programa Nacional de Imunização (PNI) com o objetivo de coordenar as ações de imunização de forma organizada e estruturada às demais estratégias de saúde pública. Inicialmente, o programa contemplava cinco vacinas: BCG, DTP (difteria, tétano, coqueluche), sarampo, poliomielite e antivariólica(1).

Atualmente, o Brasil é um dos países que oferece o maior número de vacinas à população, disponibilizando gratuitamente mais de 300 milhões de doses anuais sendo 43 tipos diferentes de imunobiológicos: 26 vacinas, 13 soros heterólogos (imunoglobulinas animais) e quatro soros homólogos (imunoglobulinas humanas), utilizados na prevenção e/ou tratamento de doenças.

Os resultados da cobertura da vacinação e erradicação da varíola contribuíram para o estabelecimento de uma "cultura da imunização" no país, com a continuidade da participação da sociedade em campanhas públicas contra a poliomielite, e mais recentemente, sarampo, rubéola, influenza, entre outras doenças imunopreviníveis. Iniciou-se, assim, uma nova configuração de políticas públicas que passaram a abarcar a imunização como essencial para a saúde pública da sociedade brasileira ${ }^{(9)}$.

\section{IMUNIZAÇÃO NO BR: SABERES E PRÁTICAS DA ENFERMAGEM UM OLHAR PARA A EDUCAÇÃO EM SAÚDE}

A administração de medicamentos é considerada uma das práticas mais representativas no escopo do trabalho da enfermagem. Desde que o cenário da saúde se organizou em distintas equipes profissionais, a enfermagem contribuiu com a administração de vacinas, mais precisamente a partir do século $\mathrm{XX}$, quando na década de 1980 Ihe foi atribuída, pela Lei do Exercício Profissional da Enfermagem, o preparo e a administração de medicamentos.

No entanto, conforme mencionado na abertura desse capítulo, o maior investimento da enfermagem, principalmente dos enfermeiros, em registros e pesquisas em torno das vacinas aconteceu a partir da consolidação do $\mathrm{PNI}$, corroborado por outros contextos tais como o crescimento da pesquisa nacional a partir da Reforma Universitária de 1968 e da pesquisa em enfermagem a partir do Curso de Pós-Graduação stricto sensu na área, em 1972.

Ainda que haja poucos registros na história da imunização sobre a atuação da enfermagem, considerou-se lançar luzes sobre os fatos históricos documentados, problematizados aqui em torno do trabalho 
da enfermeira de saúde pública - força de trabalho que protagonizou um novo olhar para a imunização no movimento sanitarista brasileiro.

Para tal, é importante resgatarmos que a saúde dos brasileiros não foi uma preocupação do Estado até meados do século XIX e que uma das primeiras iniciativas de intervenção marcou a história da saúde pública brasileira com a Revolta da Vacina, por ter sido conduzida de forma compulsória ${ }^{(10)}$. Assim, no início do século XX, com o sanitarismo, começou-se a buscar outras formas e profissionais que pudessem, via educação sanitária, melhorar as condições de saúde dos brasileiros que eram dizimados por doenças infecciosas, algumas delas já com vacinas disponíveis ${ }^{(11)}$.

É nesse cenário, de crise sanitária importante, que ganha força o modelo de formação em enfermagem anglo-americano, pautado nos princípios de Florence Nightingale de escolarização, a partir do diagnóstico situacional realizado por Ethel Parsons, enfermeira norte-americana, que apontou a ausência de profissionais devidamente qualificados para exercer a enfermagem no país.

Os desdobramentos da Missão Parsons são amplamente discutidos na historiografia da enfermagem brasileira ${ }^{(12-16)}$, afinal este modelo foi estruturante para a formação do enfermeiro que temos acompanhado nos últimos 100 anos. Ao pautar tal discussão, deseja-se mostrar como a enfermagem, para além da administração do imunizante, ao assumir a educação em saúde como um de seus eixos formativos, desempenhou e segue cumprindo com um papel central na mudança de paradigma em torno da vacinação na nossa sociedade.

\section{PROGRAMA NACIONAL DE IMUNIZAÇÕES E O PROTAGONISMO DA ENFERMAGEM NA IMUNIZAÇÃO}

O Programa Nacional de Imunizações (PNI), criado em 1973, consagrou-se ao longo de quase cinco décadas como uma grande conquista para a saúde pública brasileira, sendo reconhecido internacionalmente pelo processo de organização e coordenação da vacinação, a qual vem contribuindo desde o início do século XX para a redução da morbidade e mortalidade por doenças transmissíveis no Brasil ${ }^{(17-18)}$.

O PNI é reconhecido por conferir atualmente o acesso gratuito a 32 vacinas - inscritas no calendário básico de vacinação e as disponibilizadas pelos Centros de Referência de Imunobiológicos especiais. A proteção conferida se inicia nos recém-nascidos, estendendo-se por toda a vida e apresentando-se cada vez mais complexa, tanto sob o ponto de vista do aumento do quantitativo de vacinas fornecidas quanto sob a ótica da diversificação de esquemas vacinais ${ }^{(19)}$. O sucesso do PNI se deve em parte ao fato de se orientar pelos princípios do SUS, com destaque para a universalidade, a equidade da atenção e a descentralização. Assim, é coordenado pelo Ministério da Saúde, com uma gestão compartilhada junto às secretarias estaduais e municipais de saúde ${ }^{(19)}$.

Por outro lado, o Programa também se vê constantemente desafiado, dada a complexidade e dinamicidade do quadro epidemiológico, o que impõe não somente o desenvolvimento de novas vacinas, mas também a necessidade de se encontrar novos modos de organizar as ações de vacinação no âmbito nacional ${ }^{(17)}$. A pandemia da COVID-19 é um emblemático exemplo atual dos desafios impostos ao PNI e à complexidade que envolve a coordenação e organização da vacinação no Brasil.

Analisar o PNI ao longo da história e prospectá-lo a partir da complexidade epidemiológica inscrita no cenário atual é introduzi-lo em um lugar de consolidação de conquistas já alcançadas e de enfrentamentos necessários, dado o complexo perfil epidemiológico das doenças transmissíveis em um mundo no qual os riscos sanitários são compartilhados de maneira quase imediata pelas populações ${ }^{(18)}$.

Além disso, analisar o PNI ao longo da história é também considerar o protagonismo de diversos atores no campo da gestão, da vigilância em saúde e das ações que envolvem diretamente a prática da vacinação. Entre estes atores dá-se destaque para os inscritos na Enfermagem, os quais ao longo da trajetória do PNI tem escrito também a história da profissão no contexto da imunização das populações. 
A Resolução do Conselho Federal de Enfermagem (COFEN) n.302, de 16 de março de 2005, estabelece os contornos desse exercício profissional. Nesta consta que cabe ao enfermeiro orientar e prestar uma assistência em condições seguras aos usuários dos serviços de saúde, assumindo a responsabilidade pela conservação e controle dos imunobiológicos, acompanhamento das doses administradas e investigação de possíveis efeitos adversos. Ademais, cabe ao enfermeiro realizar junto à equipe uma educação continuada e permanente, de modo que as melhores evidências científicas sejam incorporadas às práticas de imunização(20).

Portanto, o PNI se materializa cotidianamente nos serviços de saúde com a presença e atuação fundamental da Enfermagem, especialmente do enfermeiro, que é o responsável técnico pelas atividades concernentes à imunização ${ }^{(21)}$. Nesta perspectiva, a vacinação coloca o enfermeiro na centralidade das ações engendradas no PNI, considerando ser este o profissional habilitado para supervisionar e atuar diretamente nas etapas e procedimentos relacionados a tal prática no cotidiano do SUS.

Diante do exposto, propomos estabelecer um diálogo da atuação da Enfermagem no processo de consolidação do PNI, buscando uma interface desta prática com as dimensões política, gerencial, assistencial e educativa do cuidar em saúde.

Considerando ser o PNI uma importante política pública de inclusão social, as ações produzidas para a sua materialização na vida dos usuários do SUS se configuram como ações políticas. O enfermeiro, neste sentido, exerce a politicidade do cuidado no bojo do PNI, pois com a sua prática social provoca transformações significativas no perfil epidemiológico da população, atuando na vigilância e prevenção de doenças imunopreviníveis.

A dimensão gerencial do cuidado em Enfermagem se inscreve no PNI nos diversos espaços ocupados e ações realizadas pela Enfermagem, envolvendo desde o gerenciamento macro desta política até os microprocessos que acontecem para o funcionamento adequado das salas de vacina existentes no Brasil no âmbito do SUS.

Tal gerenciamento impõe à Enfermagem a necessidade de organizar, planejar, executar e avaliar com conhecimento e segurança a prática da vacinação, de modo que o imunobiológico administrado provoque a resposta imune necessária em indivíduos e coletividades, atuando efetivamente no controle e/ou erradicação de doenças ${ }^{(22)}$. No contexto atual da pandemia da COVID-19, o enfermeiro assumiu o preponderante papel de gestor na preparação da sua unidade de saúde e de todas as medidas relacionadas à vacinação COVID 2021.

Entremeada ao gerenciamento inscreve-se também a dimensão assistencial do cuidado. Esse é o lugar de maior visibilidade da Enfermagem no contexto do $\mathrm{PNI}$, pois diz respeito ao momento em que o direito à vacinação alcança cada brasileiro e brasileira. A Enfermagem, que está à frente das 38 mil salas de vacina no serviço público de saúde do Brasil, precisa exercer com excelência a sua prática profissional. Neste contexto inscrevem-se os conhecimentos, habilidades e atitudes necessárias à prática da imunização, que compreende desde a conservação e controle adequados dos imunobiológicos na sala de vacina até o acompanhamento de eventos pós vacinais ${ }^{(23)}$.

Os registros são importantes aspectos a serem considerados na dimensão assistencial da prática da vacinação, tanto no que tange à possibilidade de acompanhamento da situação vacinal pelos próprios usuários - por meio do cartão de vacina - quanto no tocante a gerar informações em saúde, por meio do Sistema de Informação do Programa Nacional de Imunizações (SI PNI). Este, de modo particular, permite avaliar as coberturas com maior acurácia e identificar o indivíduo vacinado ${ }^{(24)}$, propondo ser uma ferramenta importante para o trabalho da enfermagem. Assim, o enfermeiro que dela se apropria consegue melhor avaliar as práticas relacionadas à vacinação, identificando fragilidades, melhorias e o planejamento/tomada de decisões necessários no cotidiano dos serviços ${ }^{(21)}$.

As boas práticas de enfermagem no contexto do PNI envolvem também a necessidade de garantir a exposição dos usuários ao menor risco possível. Neste contexto dá-se destaque às ações da farmacovigilância, incluindo-se os eventos adversos pós-vacinação (EAPV), nas quais a atuação do enfermeiro torna-se fundamental ${ }^{(25)}$. A Enfermagem tem se debruçado no conhecimento sobre os EAPV, de modo a reduzir a incidência 
de eventos evitáveis e, por conseguinte proporcionar maior segurança e confiabilidade do Programa por parte da população.

A dimensão educativa do cuidado de enfermagem assume também um lugar fundamental e privilegiado no campo da imunização, pois figura como uma estratégia transformadora das práticas de saúde tecidas cotidianamente no bojo do PNI. Neste sentido, a competência educativa se revela como uma potência na atuação do enfermeiro e da enfermagem, atravessando diferentes contextos e ações inscritas no Programa.

A educação em saúde estimula a efetiva participação e o envolvimento da família no processo que envolve a aplicação de imunobiológicos. O enfermeiro e a equipe de enfermagem, por meio da sua capacidade de atuação territorial, têm a potencialidade de estabelecer vínculo com a família e, consequentemente, desenvolver ações de educação e promoção em saúde, com ênfase para o esclarecimento de dúvidas e cuidados relacionadas à vacina, esquemas de vacinação e importância das mesmas. Ademais, a dimensão educativa do cuidar em enfermagem se faz presente no enfermeiro que assume desde a gestão/coordenação das ações no âmbito das secretarias estaduais e municipais de saúde até os que atuam nas milhares de salas de vacina do Brasil, considerando a necessidade e importância da educação permanente e continuada no âmbito da vacinação.

Destarte, depreende-se que a imunização requer reflexão cotidiana pelo enfermeiro e, equipe de enfermagem, desvelando o chamamento para a Educação Permanente (EP) em saúde, considerada desafiadora e necessária. A complexidade e dinamicidade do trabalho em sala de vacina pressupõem a realização de atividades educacionais que vislumbrem o desenvolvimento dos trabalhadores e a consequente melhoria na qualidade da atenção à saúde das populações ${ }^{(26)}$.

Ao pautar a educação em saúde faz-se necessário compreender que educar envolve a comunicação, que se constitui uma competência básica na formação em enfermagem e na atuação da profissão. Assim, o enfermeiro, no cotidiano da vacinação, precisa se valer de uma comunicação efetiva, quiçá terapêutica ${ }^{(27)}$, de modo que os conhecimentos técnico-científicos incorporados neste campo de atuação sejam validados pelos usuários do SUS. No ato de educar em saúde na vacinação o enfermeiro/a enfermagem comunica com as pessoas a serem vacinadas, com pais e/ou responsáveis, líderes comunitários, veículos de comunicação de massa, entidades científicas, entre outras, de modo a ampliar e fortalecer estratégias que aumentem a adesão da população(22).

Nessa perspectiva, fortalecer estratégias de educação e comunicação eficazes torna-se cada vez mais importante, já que enfrentamos atualmente a queda e a enorme heterogeneidade na cobertura vacinal entre os municípios e estados brasileiros ${ }^{(1)}$. Tal fato tem configurado um grande desafio do PNI na atualidade, sendo a hesitação vacinal - indecisão em aceitar as recomendações das vacinas disponíveis nos serviços de saúde, levando a atraso ou recusa da vacinação - uma das maiores preocupações desde 2016, ano em que o Brasil começou a apresentar quedas substanciais das coberturas vacinais ${ }^{(28)}$.

O desafio inscrito na queda das coberturas vacinais posto ao PNI se descortina como um desafio também direcionado ao enfermeiro/ equipe de enfermagem. Estes, que têm se revelado como pilares do Programa há aproximadamente meio século, são atualmente chamados a encontrar estratégias para que doenças imunopreviníveis não voltem a assombrar a população brasileira.

Que o educar, assistir, gerenciar e atuar politicamente sejam traduzidos como armamento de cuidado aos usuários do SUS diante dos enfrentamentos necessários à Enfermagem no contexto do PNI, assumindo como premissa nosso agenciamento a um direito à saúde: a vacinação.

\section{CONSIDERAÇÕES FINAIS}

Até aqui, vimos que a história das vacinas é antiga. Sem os imunobiológicos, faltaria um pilar da civilização contemporânea, já que nos assombraria um índice de mortalidade infantil alto, uma baixa expectativa de vida, crescimento demográfico estagnado e atrasos sociais e tecnológicos. 
No ano de 2020, o mundo iniciou o enfrentamento da pandemia de COVID-19, mesmo ano em que a enfermagem vivenciava a Campanha Nursing Now, cujo objetivo principal era apresentar os profissionais da enfermagem como os exímios protagonistas da saúde brasileira ${ }^{(29)}$. Além disso, esse ano foi dedicado a rememoração das ideias de Florence Nightingale pela passagem dos 200 anos de seu nascimento, que colocou no mundo aquela que seria a responsável por elevar o papel da enfermagem mundialmente na assistência em saúde. A pandemia possibilitou a visibilização da relevância dos cuidados de Enfermagem para a manutenção da vida e o direito à saúde, as comemorações evocando Nightingale reavivaram práticas de higiene por ela incentivadas como a lavagem das mãos e a Campanha corroborou o chamado para valorização dos profissionais de enfermagem ${ }^{(30)}$.

Embora a realidade imposta pela pandemia por SARS-CoV-2, evidencie a importância do papel da enfermagem no processo de cuidar, desvelou-se também as condições de trabalho precarizadas às quais os trabalhadores da saúde estão expostos ${ }^{(31)}$. Tal fato reflete na saúde do trabalhador e consequentemente na segurança do paciente, uma vez que as condições de trabalho atual trazem o adoecimento dos profissionais, o funcionamento inadequado dos serviços assistenciais e a dificuldade na proteção dos pacientes ${ }^{(30)}$. Assim, valorizar o trabalho da enfermagem reflete diretamente na qualidade dos serviços de saúde prestados à sociedade.

Entendendo a representatividade que os profissionais da enfermagem têm no contexto da pandemia da COVID-19, no dia 17 de janeiro de 2021, após a aprovação do uso emergencial de vacinas pela Anvisa (Agência Nacional de Vigilância Sanitária), a enfermeira Mônica Calazans foi a primeira pessoa a ser vacinada contra a COVID-19 no Brasil.

Mônica Calazans, mulher de 54 anos, negra, enfermeira intensivista e atuante na linha de frente de combate à COVID-19 desde o início da pandemia, recebeu o imunizante Coronavac, desenvolvido no Brasil pelo Instituto Butantan em parceria com o laboratório chinês Sinovac, no Hospital das Clínicas de São Paulo. A enfermeira apresenta perfil de alto risco para complicações da COVID-19, devido ao quadro de obesidade, diabetes e hipertensão. Mas, independentemente disso, ela desenvolve suas atividades laborais em uma unidade de saúde que está no epicentro do enfrentamento da pandemia.

A enfermeira Mônica Calazans foi a vencedora do prêmio Notáveis CNN (Cable News Network) em 2020 evidenciando a sua luta contra o novo coronavírus. Ser a primeira pessoa vacinada contra a COVID 19 no país e, ser reconhecida pela sua história no combate a essa doença desvela a real situação da categoria profissional da enfermagem nesse cenário. Afinal, o maior contingente de profissionais que estão na linha de frente do cuidado ao paciente com COVID-19 é o de enfermagem o que torna esses profissionais os mais vulneráveis à doença ${ }^{(32)}$.

\section{REFERÊNCIAS}

1. Homma A, Possas C, Noronha JC, Gadelha P, organizadores. Vacinas e vacinação no Brasil: horizontes para os próximos 20 anos [Internet]. Rio de Janeiro: Edições Livres; 2020 [cited 2021 Jun 02]. Available from: https://www.arca.fiocruz.br/ bitstream/icict/45003/2/Livro\%20Vacinas\%20no\%20Brasil-1.pdf

2. Buss PMI, Temporão JG, Carvalheiro JR, organizadores. Vacinas, soros \& imunizações no Brasil [Internet]. Rio de Janeiro: Editora Fiocruz; 2005 [cited 2021 May 20]. Available from: http://books.scielo.org/id/wmw76

3. Feijó RB, Sáfadi MA. Immunizations: three centuries of success and ongoing challenges. J Pediatr (Rio J). 2006;82(Suppl3):S1-3. https://doi.org/10.2223/JPED.1497

4. Moura Filho EA. Os imunobiológicos na proteção da saúde: conhecendo sua história. In: Silva MN, Flauzino RF, Gondim GMM, editores. Rede de frio: fundamentos para a compreensão do trabalho. Rio de Janeiro: Editora FIOCRUZ;2017. https:// doi.org/10.7476/9786557080917.0010

5. World Health Assembly, 52. Smallpox eradication: destruction of variola virus stocks: report by the Secretariat [Internet]. Geneva:WHO; 1999 [cited 2021 May 18]. Available from: https://apps.who.int/iris/handle/10665/78118 
6. Stevanim LF. Uma vacina para a humanidade. Rev Radis [Internet]. ENSP: 2020 [cited 2021 May 18]. Available from: https:// radis.ensp.fiocruz.br/phocadownload/revista/Radis216_web.pdf

7. Programa das Nações Unidas para o Desenvolvimento. Os Objetivos de Desenvolvimento Sustentável [Internet]. Brasil: PNUD; 2015 [cited 2021 Mar 28]. Available from: http://www.pnud.org.br/ods.aspx

8. Teixeira LA. A trajetória do Instituto Butantan: pesquisa e produção de imunobiológicos para a saúde pública. Rev Bras Inov [Internet]. 2016 [cited 2021 Jun 02];15(1):165-74, Available from: https://periodicos.sbu.unicamp.br/ojs/index.php/rbi/ article/view/8649124/15673

9. Hochman G. Vaccination, smallpox, and a culture of immunization in Brazil. Ciênc Saúde Colet. 2011;16(2):375-86. https:// doi.org/10.1590/S1413-81232011000200002

10. Moutinho FFB. Conflitos da sociedade brasileira com as normas sanitárias: um paralelo entre a revolta da vacina e a pandemia de COVID-19. Hygeia-Rev Bras Geogr Méd Saúde. 2020:60-71. https://doi.org/10.14393/Hygeia0054392

11. Finkelman J, organizador. Caminhos da saúde pública no Brasil [Internet]. Rio de Janeiro: Editora Fiocruz; 2002 [cited 2021 Jun 02]. 328p. Available from: https://www.arca.fiocruz.br/bitstream/icict/2705/1/Finkelman_Jacobo(Org.).pdf

12. Sauthier J, Carvalho V. A Missão Parsons: documentos históricos da EEAN. UFRJ-1922-1931. Rio de Janeiro: Anna Nery; 1999.

13. Barreira IA. A reconfiguração da prática da enfermagem brasileira em meados do século 20. Texto Contexto Enferm. 2005;14(4). https://doi.org/10.1590/S0104-07072005000400003

14. Mancia JR, Padilha MICS. La trayectoria de Edith Magalhães Fraenkel. Rev Bras Enferm. 2006;59(spe). https://doi. org/10.1590/S0034-71672006000700009

15. Peters AA, Peres MAA, D'antonio P. Influences of the Anglo-American Teaching System in Brazil: Contributions by the Parsons Mission (1921-1925). OJIN: Online J Iss Nurs. 2020;25(2). https://doi.org/10.3912/OJIN.Vol25No02Man06

16. Korndörfer AP, Ramacciotti KI. Uma proposta, duas experiências: a Fundação Rockefeller e a formação de enfermeiras no Brasil e na Argentina (primeira metade do século XX). Descentrada. 2021;5(1):e131. https://doi. org/10.24215/25457284e131

17. Silva Jr JB. 40 anos do Programa Nacional de Imunizações: uma conquista da Saúde Pública brasileira. Epidemiol Serv Saúde. 2013;22(1):7-8. https://doi.org/10.5123/S1679-49742013000100001

18. Domingues CMAS, Maranhão AGK, Teixeira AM, Fantinato FFS, Domingues RAS. The Brazilian National Immunization Program: 46 years of achievements and challenges. Cad Saúde Pública. 2020;36(2):e00222919. https://doi. org/10.1590/0102-311X00222919

19. Domingues CMAS, Teixeira AMS. Coberturas vacinais e doenças imunopreveníveis no Brasil no período 1982-2012: avanços e desafios do Programa Nacional de Imunizações. Epidemiol Serv Saúde. 2013;22(1):9-27. https://doi.org/10.5123/ S1679-49742013000100002

20. Conselho Federal de Enfermagem (Cofen). Resolução COFEN n. 302/2005 [Internet]. São Paulo: Cofen; 2005 [cited 2021 Jun 03]. Available from: http://www.cofen.gov.br/resoluo-cofen-3022005_4337.html

21. Silva BS, Coelho HV, Cavalcante RB, Oliveira VC, Guimarães EAA. Evaluation study of the National Immunization Program Information System. Rev Bras Enferm. 2018;71(Suppl1):615-24. https://doi.org/10.1590/0034-7167-2017-0601

22. Leite JCA. O "saber", o "fazer" e o "ser" enfermeiro nas práticas de imunização. In: Souza CMRM, Horta NC, organizadores. Enfermagem em Saúde Coletiva: Teoria e Prática. Rio de Janeiro: Guanabara Koogan; 2013. p.157-190.

23. Ministério da Saúde (BR). Secretaria de Vigilância em Saúde. Departamento de Vigilância das Doenças Transmissíveis. Manual de Normas e Procedimentos para Vacinação [Internet]. Brasília: Ministério da Saúde; 2014 [cited 2021 Jun 22 ]. Available from: https://bvsms.saude.gov.br/bvs/publicacoes/manual_procedimentos_vacinacao.pdf

24. Ministério da Saúde (BR). Datasus. SIPNI - Sistema de Informações do Programa Nacional de Imunizações [Internet]. 2017 [cited 2021 Apr 15]. Available from: http://datasus.saude.gov.br/sistemas-e-aplicativos/epidemiologicos/si-pni

25. Bisetto LHL, Cubas MR, Malucelli A. Nursing practice in view of adverse events following vaccination. Rev Esc Enferm USP. 2011;45(5):1128-34. https://doi.org/10.1590/S0080-62342011000500014

26. Martins JRT, Alexandre BGP, Oliveira VC, Viegas SMF. Permanent education in the vaccination room: what is the reality?. Rev Bras Enferm. 2018;71(Suppl 1):668-76. https://doi.org/10.1590/0034-7167-2017-0560

27. Maftum MA, Stefanelli MC. O uso das técnicas de comunicação terapêutica na relação interpessoal com o doente mental. Cogitare Enferm. 2000;5(2):69-74. https://doi.org/10.5380/ce.v5i2.44889 
28. Sato APS. What is the importance of vaccine hesitancy in the drop of vaccination coverage in Brazil? Rev Saude Publica. 2018;52:96. https://doi.org/10.11606/S1518-8787.2018052001199

29. Cassiani SHB, Lira Neto JCG. Nursing Perspectives and the “Nursing Now” Campaign. Rev Bras Enferm. 2018;71(5):2351-2. https://doi.org/10.1590/0034-7167.2018710501

30. Oliveira KKD, Freitas RJM, Araújo JL, Gomes JGN. Nursing Now e o papel da enfermagem no contexto da pandemia e do trabalho atual. Rev Gaúcha Enferm. 2021;42(esp):e20200120. https://doi.org/10.1590/1983- 1447.2021.20200120

31. Vedovato TG, Andrade CB, Santos DL, Bitencourt SM, Almeida LP, Sampaio JFS. Trabalhadores(as) da saúde e a COVID-19: condições de trabalho à deriva?. Rev Bras Saude Ocup. 2021;46(1). https://doi.org/10.1590/2317-6369000028520

32. Renast. Boletim CoVida: pandemia de COVID-19: a saúde dos trabalhadores de saúde no enfrentamento da pandemia da COVID-19 [Internet]. 2020 [cited 2021 Jun 14]. Available from: http://renastonline.ensp.fiocruz.br/sites/default/files/ arquivos/recursos/boletim-covida-5-trabalhadores-da-saude.pdf 